\title{
Study of Wavelet Methodology and Chaotic Behavior of Produced Particles in Different Phase Spaces of Relativistic Heavy Ion Collisions
}

\author{
M. Ayaz Ahmad' ${ }^{1}$, Vyacheslav V. Lyashenko ${ }^{2}$, Zhanna V. Deineko ${ }^{3}$, \\ Jalal Hasan Baker1, Shafiq Ahmad ${ }^{4}$
}

${ }^{1}$ Physics Department, Faculty of Science, University of Tabuk, Tabuk, Saudi Arabia

${ }^{2}$ Department of Informatics, Kharkiv National University of Radio-Electronics, Kharkiv, Ukraine

${ }^{3}$ Department of Media Systems and Technology, Kharkiv National University of Radio Electronics, Kharkiv, Ukraine

${ }^{4}$ Department of Physics, Aligarh Muslim University, Aligarh, India

Email: mayaz.alig@gmail.com, lyashenko.vyacheslav@gmail.com,dgv_zhanna@ukr.net, sahmad2004amu@yahoo.co.in

How to cite this paper: Ahmad, M.A., Lyashenko, V.V., Deineko, Z.V., Baker, J.H. and Ahmad, S. (2017) Study of Wavelet Methodology and Chaotic Behavior of Produced Particles in Different Phase Spaces of Relativistic Heavy Ion Collisions. Journal of Applied Mathematics and Physics, 5, 1130-1149.

https://doi.org/10.4236/jamp.2017.55100

Received: February 19, 2017

Accepted: May 23, 2017

Published: May 27, 2017

Copyright $\odot 2017$ by authors and Scientific Research Publishing Inc. This work is licensed under the Creative Commons Attribution International License (CC BY 4.0).

http://creativecommons.org/licenses/by/4.0/

\begin{abstract}
We applied the wavelet methodology for our earlier published research work of the chaotic behavior so called multiplicity fluctuations of secondary charged particles produced during the nucleus-nucleus (A-A) collisions at an energy of the order of $\approx 409 \mathrm{GeV}$ in a new fashion. We illustrated the wavelet coherency in a relation of chaotic behavior for above said data of secondary charged pions in different phase spaces of collisions such as: $\eta$-space, $\phi$-space (in one dimension) and $\eta \phi$-space (in two dimensions) respectively. We have shown the changes in the wavelet coherence when there are different values of two parameters "q" and "p". We discussed our new results for the comparison purpose and findings were in the good agreements.
\end{abstract}

\section{Keywords}

Wavelet Coherence, Morlet Wavelet, Dynamics of Nucleus-Nucleus (A-A)

Collisions, Phase Space, Normalized Factorial Moments (SFMs)

\section{Introduction}

The main objective of relativistic heavy ion collisions lies in the investigating of properties of nuclear matter consisting of strongly interacting particles at extreme condition of temperature and density. The most important theory from strongly interacting matter i.e. quantum chromodynamics (QCD) [1] [2] suggests that at a critical temperature of approximately $200-250 \mathrm{MeV}$, a phase 
transition from hadronic matter to a novel state of matter that is quark gluon plasma (QGP) occurred which consists of the deconfined quarks and gluons with in a small volume. The energy density for this new phase transition should be a few $\mathrm{GeV} / \mathrm{fm}^{3}\left(\sim 2 \mathrm{GeV} / \mathrm{fm}^{3}\right)$ compared with $0.17 \mathrm{GeV} / \mathrm{fm}^{3}$ of normal nuclear matter in its ground state [3] [4]. Observations of such type of phase transition would confirm the predictions of QCD and it is also of prime importance in Astrophysics. According to Big Bang theory the initial state of matter consists of dense and hot plasma of unconfined quarks and gluons: after that cosmological expansion process takes place and the energy density decreases, the plasma cools down and transforms into colorless objects [1] [2] [3] [4] [5]. This process is called harmonization. Therefore one can expect that the observation of quarks-gluon plasma (QGP) would reproduce in laboratory [5], the hypothetical state of matter that prevailed in early universe. According to phase diagram shown in Figure 1 of strongly interacting matter following points may be noted about the newly state of matter (QGP) [6].

(i) Low temperature and high nuclear energy density is believed to have existed in the early Universe and today it may exist in the heart of neuron star. One can achieve it directly.

(ii) High temperature and low nuclear energy density, which is applicable only by relativistic heavy ion collisions experiments in laboratory which is called Little Bang i.e. Relativistic Heavy Ion Collisions (RHIC) and Large Hadron Collider (LHC) and so many future experiments.

Already now there are plenty of studies that describe different experiments. In references [7] [8] showed that the high energy nucleus-nucleus (A-A) collisions provide a system in which the properties of hot, dense strongly interacting matter can be achieved. Also there have been numerous experimental results, which indicate that collisions of hadron-nucleus (h-A), nucleus-nucleus (A-A) interactions

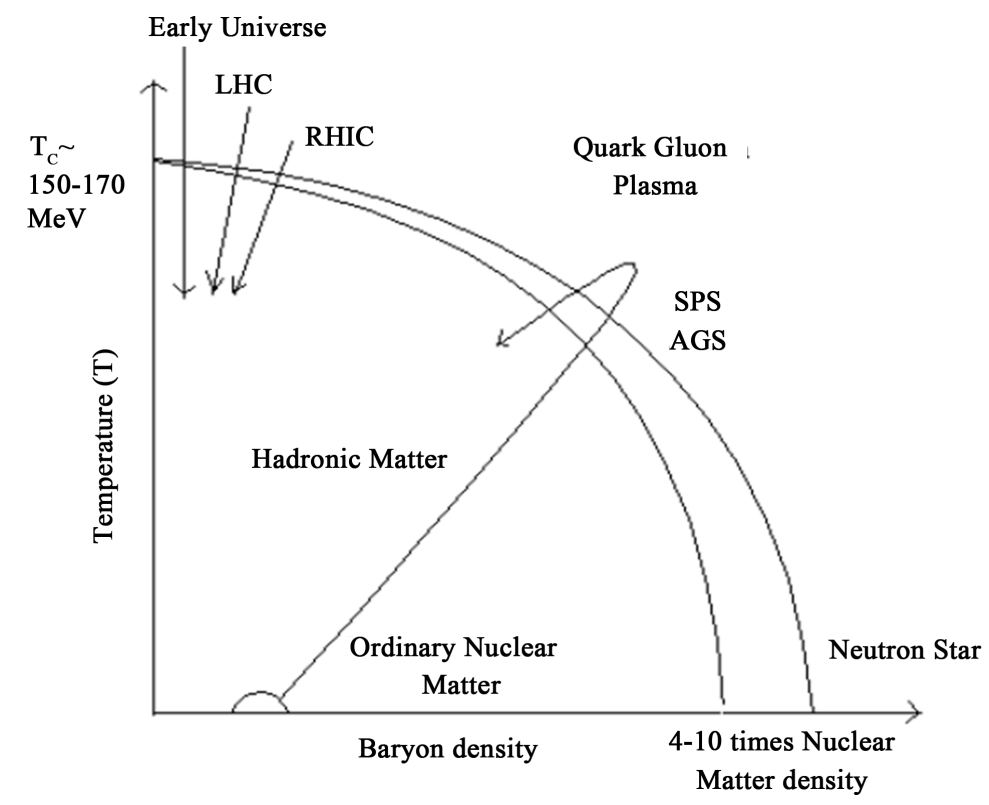

Figure 1. A schematic phase diagram of strongly interacting matter [6]. 
cannot be completely understood in term of superposition of nucleon-nucleon scattering [9] [10] [11]. There are many studies where such experimental results have been compared with the data generated with the computer code FRITIOF based on Lund Monte Carlo Model for high energy nucleus-nucleus collisions [12] [13]. The measured phenomena, such as strangeness enhancement and $J / \psi$ suppression show that extremely dense strongly interacting matter has been found [14]. But still of such research is not enough in order to completely understand the nature of heavy ion collisions at ultra-relativistic energies.

One significant area of research is the study of the dynamics of the chaotic behavior in nuclear collisions at ultra-high energies. Here it is necessary to understand such things: the accuracy of the estimates of the chaotic behavior in relativistic heavy ion collisions in different phase spaces and the connection of such assessments with each other. These questions and is dedicated to present study. To solve this problem we use wavelets ideology [15] [16]. This is due to the fact that of the chaoticity and/or errticity pattern in ultra-relativistic heavy ion collisions can have quite difficult structure, contain local features of the various form and time extent.

Finally, In this article, an attempt has been made to study the wavelet methodology over a chaotic system to understand the underlying relation of the chaotic behavior of relativistic shower particles produced in ${ }^{28} \mathrm{Si}$-emulsion interactions at $14.6 \mathrm{AGeV}$. This will help to extract the valuable information's about the mechanism of multi-particle production in different phase spaces; $\eta$-space, $\phi$-space (in one dimension) and $\eta \phi$-space (in two dimensions). The merits of wavelet analysis over chaotic behavior were foundto be in good agreements.

\section{Brief Detail of Experimental Data}

The track detectors (so called nuclear emulsion detector) have been extensively used in high energy physics over many decades. This nuclear emulsion detector also called $4 \pi$ detector and used for recording of secondary charged particles and is accompanied by the emergence of observable traces (track producing particles) corresponding to the elementary particle trajectories. Therefore in the present experiment, a FUJI film detector has been used to pick the data samples. In this experiment a beam(projectile) of ${ }^{28} \mathrm{Si}$ nuclei at total energy $\approx 409 \mathrm{GeV}$ hit to the heterogeneous mixture/various nuclei (fixed target) of nuclear emulsion at Alternating Gradient Synchro-phasotron (AGS) of Brookhaven National Laboratory (BNL), NewYork, USA.The other relevant details about the present experiments and target identifications may be seen in our earlier publications [17]-[26]. The projectile spectator fragments, singly and multiple charged, emitted inside fragmentation cone, usually we determine the number of alpha particles $\left(n_{\alpha}\right)$ and the number of fragments with $Z>2$, separately [6].

The polar $(\theta)$ and azimuthal $(\phi)$ emission angles of all tracks have been measured, and the pseudo-rapidity has been calculated by $\eta=-\ln \left(\tan \left(\theta_{s} / 2\right)\right)$ for each shower particle.

This study has been carried out for the experimental data along with the 
theoretical prediction of Ultra-relativistic Quantum Molecular Dynamics model (UrQMD) and Monte-Carlo (RanMC) simulation and the total number of events/data points were 10000 .

\section{Mathematical Approach}

\subsection{Analytical Tool for Wavelet Methodology}

We made an approach for the generalization of the constant wavelet transformation over time interval " $t$ " which later on changed into an input time series such as; $x(t) \in L^{2}(R)$ with a wavelet parent $\varphi(t)$ [27] [28] [29]:

$$
W_{x}(u, s)=\int_{-\infty}^{+\infty} x(t) \frac{1}{\sqrt{s}} \varphi\left(\frac{t-u}{s}\right)
$$

where, the usual meanings to symbols such as: $\sqrt{s}$ represents to normalization processes, "u" indicates to location parameter and " $s$ " is a scale parameter.

$$
\int_{-\infty}^{\infty} \varphi(t) \mathrm{d} t=0
$$

The wavelet analysis transforms the original data to a classified structure by which we get a new set of wavelet coefficients. This is done using so-called multiresolution analysis method. The multiresolution analysis consists in splitting of an investigated number into two components-approximating and detailing, with their subsequent crushing for the purpose of change of level of expansion of a signal to the set level of expansion. The importance of wavelet analysis in the study of time series is to determine the fact that the method of wavelet analysis allows to discover the local features of the studied time series due to the decomposition of the input data [28] [29]. And finally, this allows determining the presence of special characteristics of the analyzed data, as well as the point where these characteristics may arise [30]. On the other hand, the wavelet analysis allows conducting a detailed analysis for the original data.

In practice, it is quite spread the use of discrete wavelet transformation (DWT) [15]. It is connected with that application DWT becomes especially effective when the signal has high-frequency components of short duration and extensive low-frequency components. In the consent of time series study of the wavelet analysis that, it stimulates for the various methods of wavelet transformation and their application in different sectors such as: scaled analysis, cross wavelet transformation, wavelet coherence.

But the leading role of the wavelet transformation methods is to use for generalized cross-reference analysis between different time series is wavelet coherence. The wavelet coherency simultaneously assess how the co-movement and causalities between two variables vary across different frequencies involved and change over time in a time-frequency window. Moreover, co-movement of the time series is recognizable among different time scales, which the standard approaches, failed to perform. It allows to calculate local correlation of two time series $(x$ and $y)$ in a region of time-frequency. Hence, one can use the following formalized model; e.g. the wavelet coherence as the squared absolute value of the 
smoothed cross wavelet spectra $W_{x y}(u, s)$, should normalized by the product of the smoothed individual wavelet power spectra of each series [31] [32]:

$$
R^{2}(u, s)=\frac{\left|Q\left(s^{-1} W_{x y}(u, s)\right)\right|}{Q\left(s^{-1}\left|W_{x}(u, s)\right|^{2}\right) Q\left(s^{-1}\left|W_{y}(u, s)\right|^{2}\right)}
$$

where; $Q$ is a smoothing operator.

In the present work, we used the Morlet wavelet that was a complex wavelet with good time-frequency localization, as a parent one [31] [32]. The squared wavelet coherency coefficient was in the rangeof $0 \leq R^{2}(u, s) \leq 1$, and it was found that the values close to zero indicate weak correlation, while values close to one are evidences of strong correlation.Thus, wavelet coherency analysis enables interconnection between the studied time series and analyzes the frequency of such communications. The Monte Carlo methods are used here to properly find statistical level of significance of the wavelet coherence.

\subsection{Analysis for the Chaotic Behavior}

To eliminate the effect of non-uniform density distribution, we used the proposed method of Bialas and Gazdzicki [33]. In this method the original values of pseudo-rapidity $(\eta)$ distribution is transformed into a new cumulative variable $(X(\varpi))$. And this was demonstrated by following mathematical relation:

$$
X(\varpi)=\frac{\int_{\sigma_{\min }}^{\sigma_{\max }} \rho\left(\varpi^{\prime}\right) \mathrm{d}\left(\varpi^{\prime}\right)}{\int_{\sigma_{\min }}^{\sigma_{\max }} \rho\left(\varpi^{\prime}\right) d\left(\varpi^{\prime}\right)}
$$

where the usual meanings of such above parameters were; $\rho(\varpi)=(1 / N) \mathrm{d} n / \mathrm{d} \varpi$ is the single particle $(\eta)$ distribution of the secondary charged pions and $\varpi_{\min }$ and $\varpi_{\max }$ are the two extreme points in the density distribution of $\rho(\varpi)$. The effect of this analysis the corresponding region of investigation the interval of pseudo-rapidity $\Delta \eta$, become changed in 0 to 1 order of a variable $X(\varpi)$.

Further, for the study of event-to event multiplicity fluctuations in relativistic nuclear collisions, it is necessary to investigate the performance of the event factorial foment, $F_{q}^{e}$ in small bins of variable $X(\varpi)$. The factorial moments (FMs) for the order "q" of an event was mathematically represented such as [34] [35]:

$$
F_{q}^{(e)}=\left[\frac{1}{M} \sum_{m=1}^{M} n_{m}\left(n_{m}-1\right) \cdots\left(n_{m}-q+1\right)\right] \times\left(\frac{1}{M} \sum_{m=1}^{M} n_{m}\right)^{-q}
$$

where " $M$ " is the partition number of variable $X(\varpi), n_{m}$ is the number of produced secondary charged particles into the $\mathrm{m}^{\text {th }}$ bin and $q=2-6$ is the order of the moment.

Since the fluctuations of $F_{q}^{e}$ from event-to-event, we get a distribution of $F_{q}^{e}$ denoted by $P\left(F_{q}^{e}\right)$ after a large number of events. The normalized factorial moments of a particular event can now be defined such as:

$$
\varpi_{q}(M)=\frac{F_{q}^{e}(M)}{\left\langle F_{q}^{e}(M)\right\rangle}
$$


where

$$
\left\langle F_{q}^{e}(M)\right\rangle=\frac{1}{N_{e v}} \sum_{e=1}^{N_{e v}} F_{q}^{e}(M),
$$

It is worth mentioning here that the scaled factorial moments (SFMs) defined by Bialas and Paschanski [34] [35] to study of intermittency in nuclear collisions is only an estimate of mean of the distribution $P\left(F_{q}^{e}\right)$. It should be realized that the averaging procedure, apart from its clear advantages, brings also a danger of losing some important information on spatial patterns from event-to-event. In particular, some interesting effects, if present only in a part of sample of events produced in high energy collisions, may be lost. A possible example of this kind is the quark gluon plasma (QGP), which is expected to be characterized by specific intermittency exponents [34] [35]. It is therefore, essential to investigate the full shape of the distribution and the way it changes with the bin size.

It has been argued [34]-[39] that the event-to-event fluctuations can probe the dynamics of multiparticle production more deeply than the variables such as the multiplicity distribution and the average factorial moments. Therefore, we study the chaoticity or event-to-event fluctuations in the density of particle produced in ${ }^{28} \mathrm{Si}$-emulsion collisions.

The erraticity moments, $C_{p, q}$ that quantify these fluctuations have been determined [36] [37] [38] [39] such as following relation in Equation (8). Expediency of use of this approach is discussed in the articles [36] [37] [38] [39].

$$
C_{p, q}(M)=\left\langle\varpi_{q}^{p}(M)\right\rangle=\frac{1}{N_{e v}} \sum_{e=1}^{N_{e v}} \varpi_{q}^{p}(M)
$$

where, the usual meaning of various symbols/and or parameters are such as: the " $p$ " is any positive real number, $\varpi-$ a normalized factorial moment of a single event that is associated with the component in 1 -D phase space $(\eta$-space and $\phi$ space) ([24] [25] [33] [36] and references therein] of $X(\varpi)$-variable ( $X(\varpi)$ - a variable that characterizes the number of relativistic heavy ion collisions).

\section{Results and Discussions}

\subsection{The Chaoticity in Relativistic Heavy Ion Collisions}

First we have calculated the Chaoticity, so called chaotic behavior from the present experimental data. For this task, the event factorial moments $F_{q}^{e}$ and the erraticity moments, $C_{p, q}$ were calculated for order of $q=2-4$ and parameter " $p "=\mathrm{p}=0.5,0.9,1.2,1.4$ and 1.6 by using the Equation ( 7 ) and Equation (8), the values of " $M$ ' was varied from 1 to 40 . And the outcomes of all above these calculations were represented in Figure 2, for different phase spaces; $\eta$-space, $\phi$-space (in one dimension) and $\eta \phi$-space (in two dimensions) respectively. Hence the dependence of $\ln C_{p, q}(M)$ as function of $\ln M$ in different phase spaces for the produced secondary charged particle in the collisions of ${ }^{28} \mathrm{Si}$ (projectile) with fixed target of Emulsion nuclei at energy 14.6A GeV has been depicted in Figure 2. Some part of these results already published by the author M. Ayaz Ahmad et al., in reference [40]. From the Figure 2, it can be seen that the expe- 

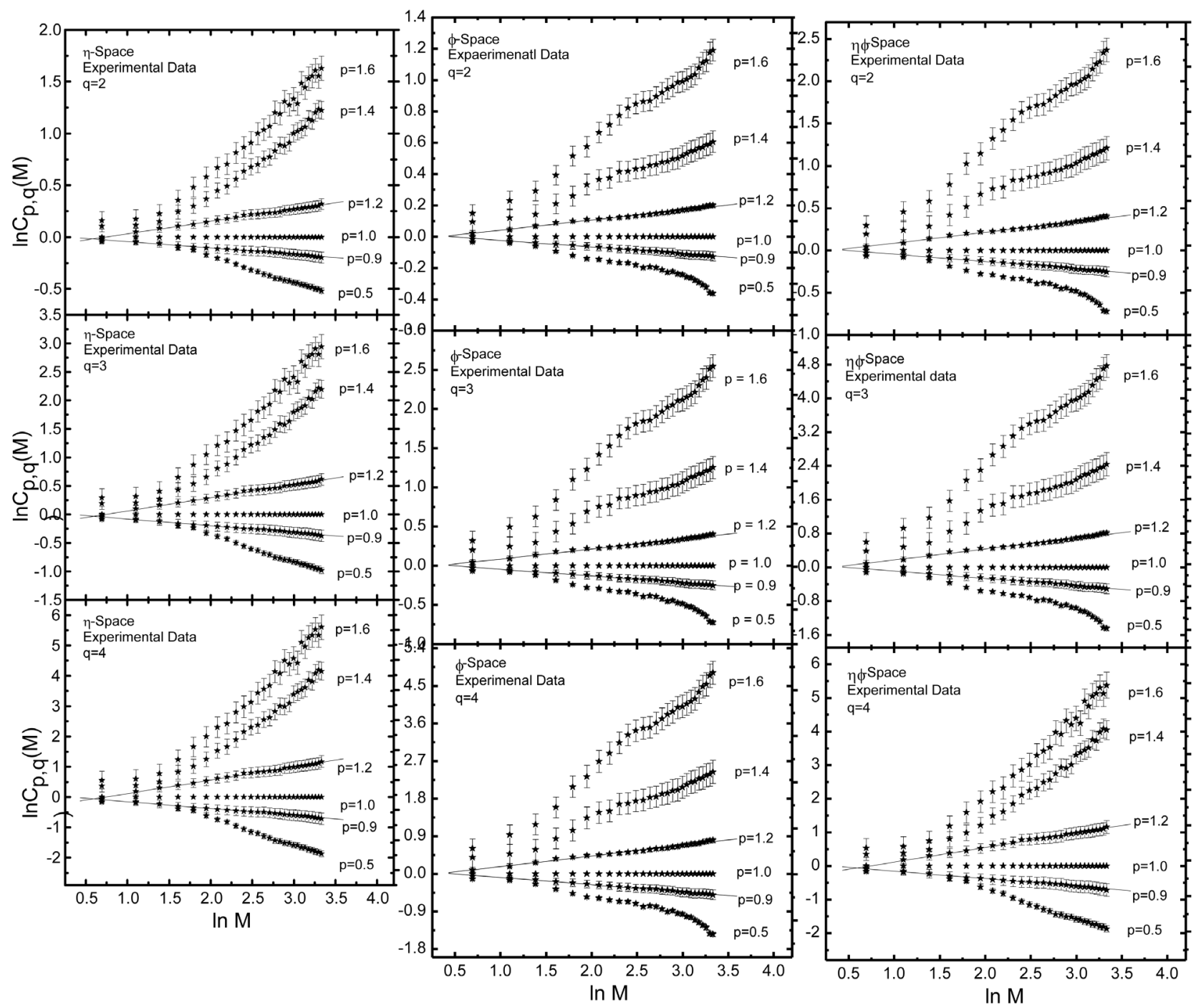

Figure 2. Variations of $\ln C_{p, q}(M)$ as function of $\ln M$ in $\eta$-space (1D) ( $\phi$-space (1D) and $\eta \phi$-space (2D)) in the collisions of ${ }^{28} \mathrm{Si}+\mathrm{Em}$ at energy $14.6 \mathrm{~A} \mathrm{GeV}$.

rimental data of $C_{p, q}$ lie well above those for the generated uncorrelated (Monte Carlo) data in the region of " $M \geq 13$. It means that the contribution of the statistical fluctuations to $C_{p, q}$ values in present experimental data is small.

Shaoshun and Zhaomin [41] and Jinghua Fu et al., [42] have studied the chaoticity in NA27 data on p-p collisions at $400 \mathrm{GeV} / \mathrm{c}$. They pointed out that the observed chaoticity could be reproduced by the statistical fluctuations only. But the chaoticity observed in the present experimental work cannot be reproduced by the statistical fluctuations only. Thus the chaotic behavior observed in our data and has the dynamical origin.

\subsection{Results from Wavelet Methodology}

Thus, we have a few of time series. These time series should be compared using the ideology of wavelets. For this important task we will use the wavelet coherency. We consider the wavelet coherency for each triple of time series for the 
produced particles in the relativistic nuclear collisions for various phase spaces; $\eta$-space, $\phi$-space (in one dimension) and $\eta \phi$-space (in two dimensions) respectively, where the factorial moments order was $q=2$ to 4 , and the parameter " $p$ " was of the order of, 0.5, 0.9, 1.2, 1.4 and 1.6.

The findings of this analysis were depicted in Figures 3-17. One can check the results of wavelet coherence between selected time series in these Figures 3-17. Each of the following figures indicated a separate group of time series that match each other. In this case the time scale (x-axis) is equal to the consistent change of values $\ln (M)$ (these changes represented a sequence number $1,2, \cdots)$. The correspondence between a sequence number and value of is shown in Table 1. In Figures 3-17 (from left to right): the first picture (a) is wavelet coherence between $\eta$-space and $\phi$-space, the second picture (b) - wavelet coherence between $\eta$-space and $\eta \phi$-space, the third picture (c) - wavelet coherence between $\phi$-space and $\eta \phi$-space.

Further it has been find that on the vertical axis were the weighted features of the analyzed data series in the frequency space. Corresponding to each of the figures there was a significant scale and is obtainable as separate columns for reflections. The wavelet coherence illustrates the regions in the time-scale space where the central variables co-vary (but do not necessarily have high power). The maximum reflection is addressed about the full coherence between the data which were analyzed. By definition, the regions inside the black lines plotted in warmer colors indicate a strong interdependence between the investigated time series. The colder color indicates relatively weak co-movement between these variables. The defined lines were a sign of localization for individual irregularities within studied time series according to importance of irregularities. The

Table 1. The correspondence between a sequence number and value of $\ln (M)$.

\begin{tabular}{cccc}
\hline Sequence number & Value of $\ln (M)$ & Sequence number & Value of $\ln (M)$ \\
\hline 1 & 0.693 & 15 & 2.773 \\
2 & 1.099 & 16 & 2.833 \\
3 & 1.386 & 17 & 2.890 \\
4 & 1.609 & 18 & 2.944 \\
5 & 1.792 & 19 & 2.996 \\
6 & 1.946 & 20 & 3.045 \\
7 & 2.079 & 21 & 3.091 \\
8 & 2.197 & 22 & 3.135 \\
9 & 2.303 & 23 & 3.178 \\
10 & 2.398 & 24 & 3.219 \\
11 & 2.485 & 25 & 3.258 \\
12 & 2.565 & 26 & 3.296 \\
13 & 2.639 & 27 & 3.332 \\
14 & 2.708 & 28 & 3.565 \\
\hline
\end{tabular}




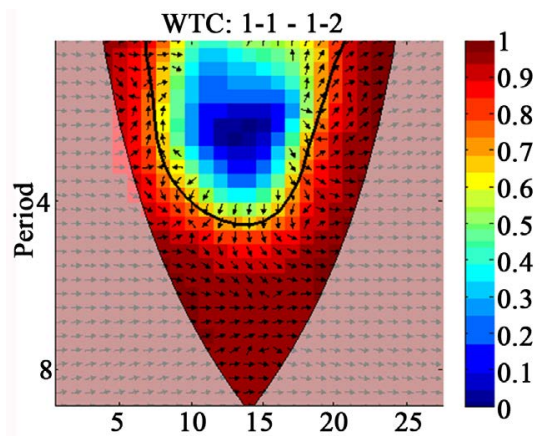

(a)

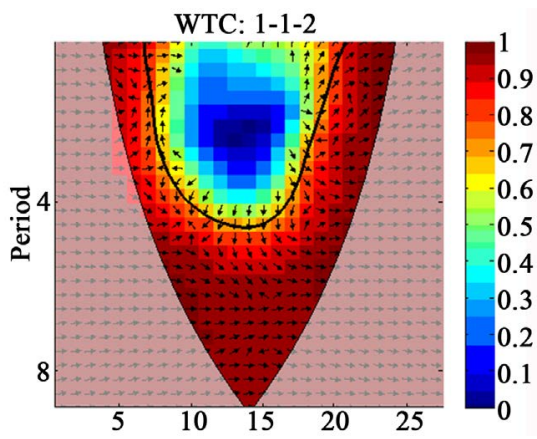

(b)

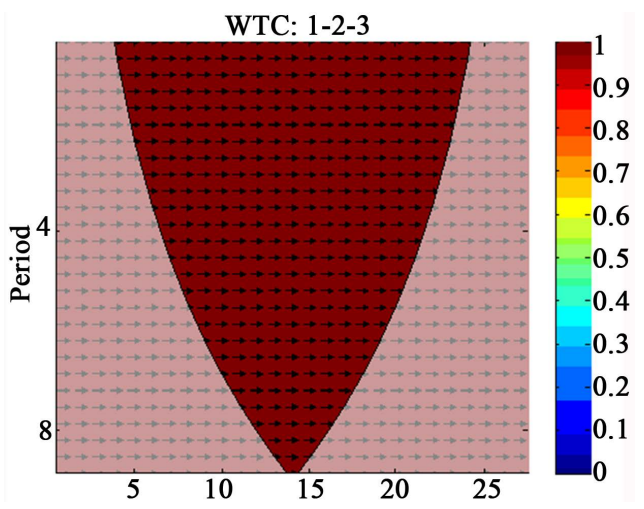

(c)

Figure 3. Wavelet coherence for the experimental data for $p=0.5$ and $q=2$.

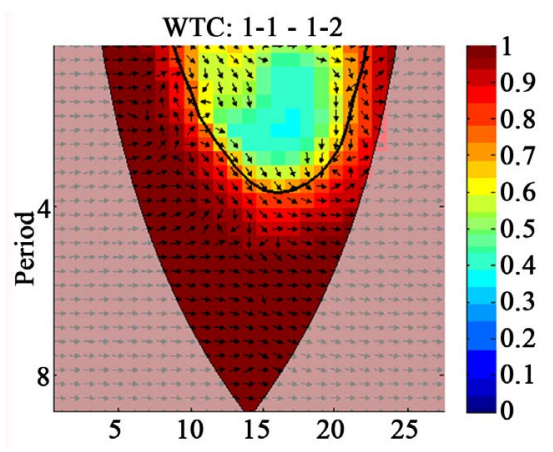

(a)

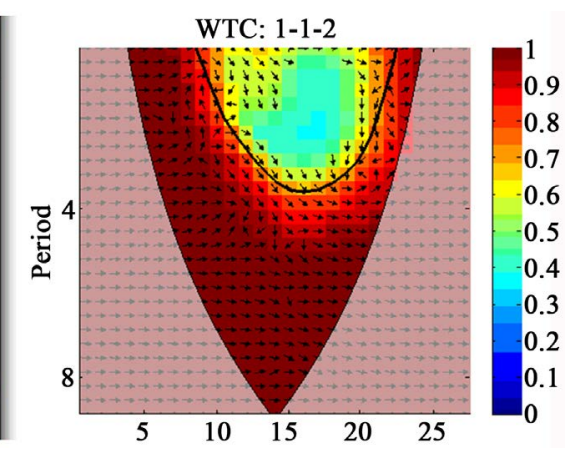

(b)

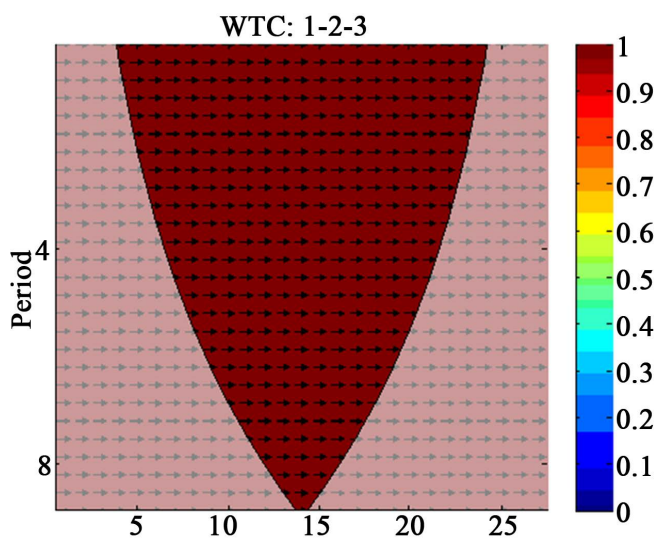

(c)

Figure 4. Wavelet coherence for the experimental data for $p=0.9$ and $q=2$. 


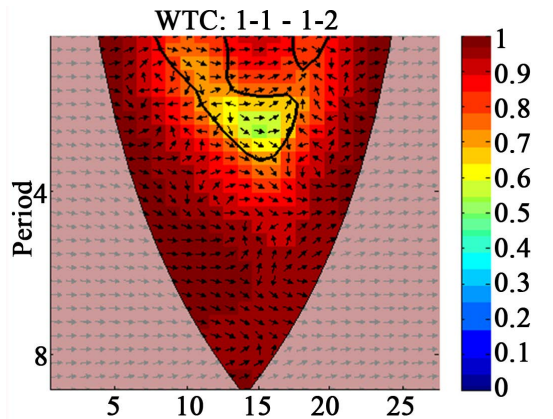

(a)

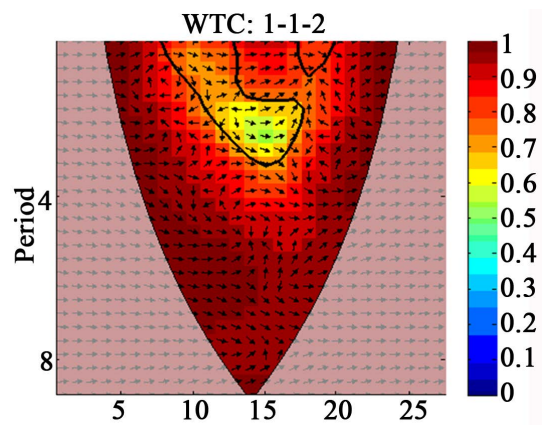

(b)

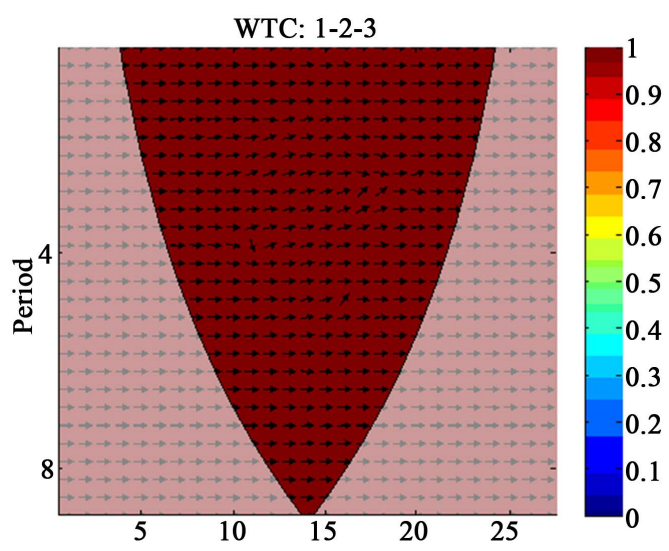

(c)

Figure 5. Wavelet coherence for the experimental data for $p=1.2$ and $q=2$.

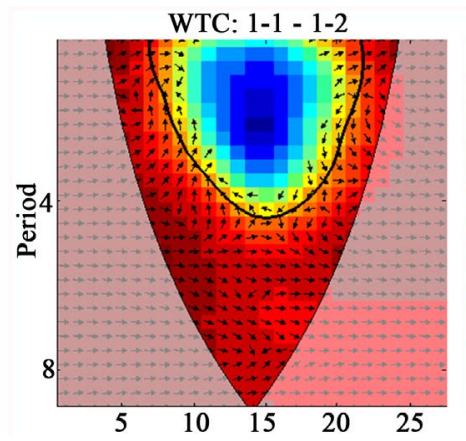

(a)

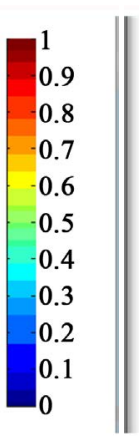

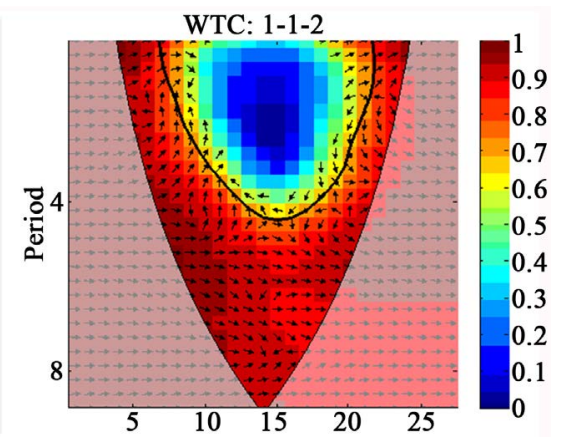

(b)

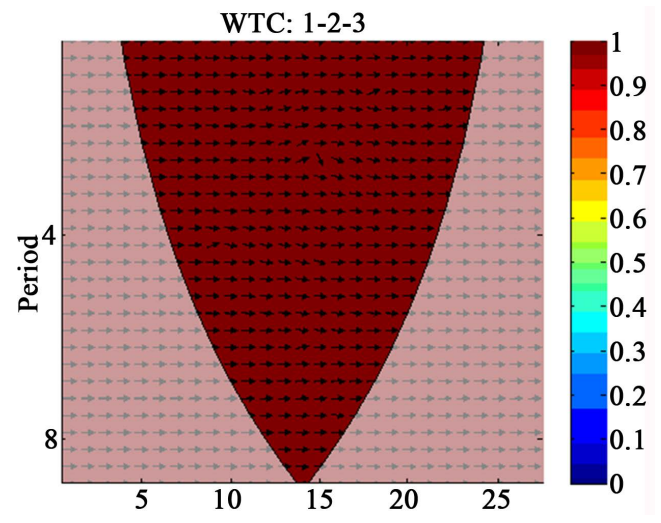

(c)

Figure 6. Wavelet coherence for the experimental data for $p=1.4$ and $q=2$. 


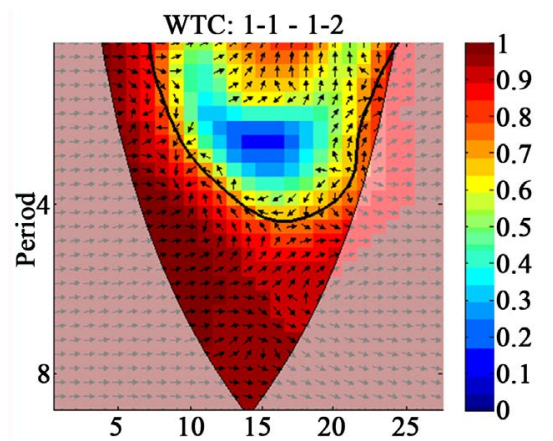

(a)

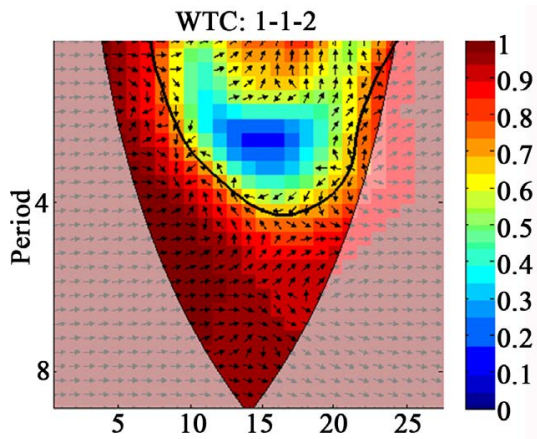

(b)

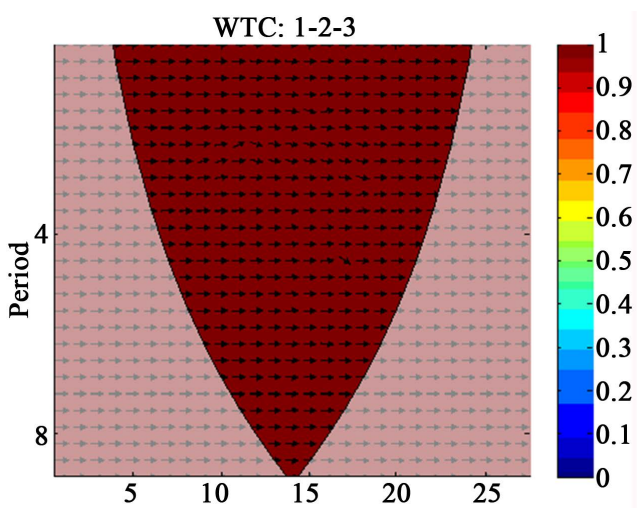

(c)

Figure 7. Wavelet coherence for the experimental data for $p=1.6$ and $q=2$.

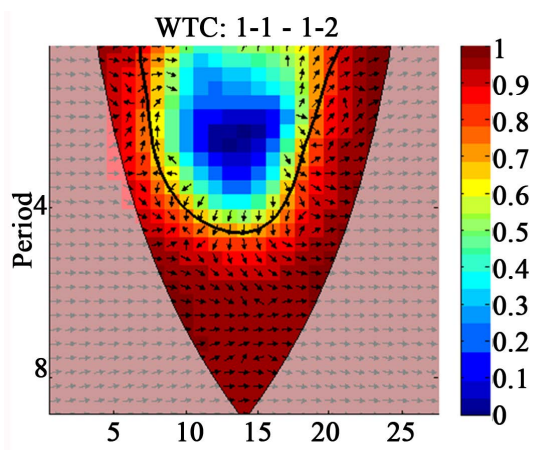

(a)

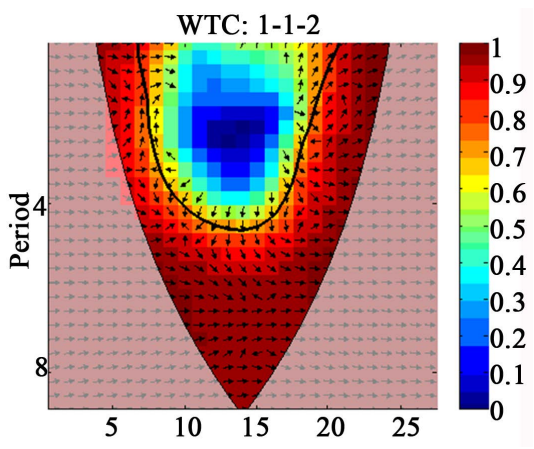

(b)

WTC: $1-2-3$

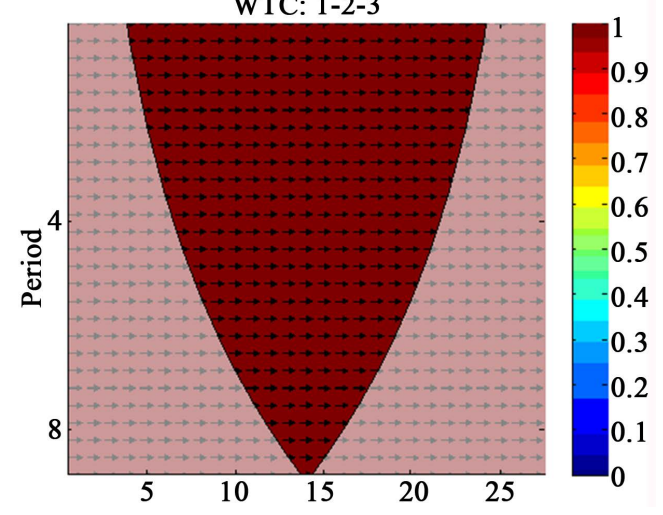

(c)

Figure 8. Wavelet coherence for the experimental data for $p=0.5$ and $q=3$. 


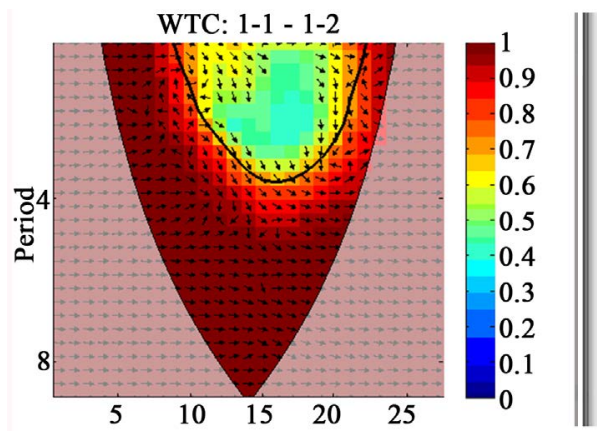

(a)

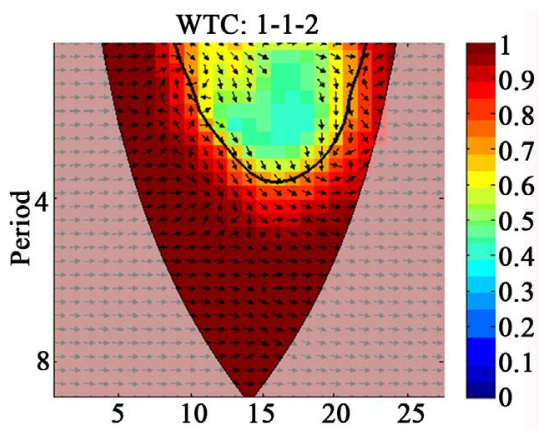

(b)

WTC: $1-2-3$

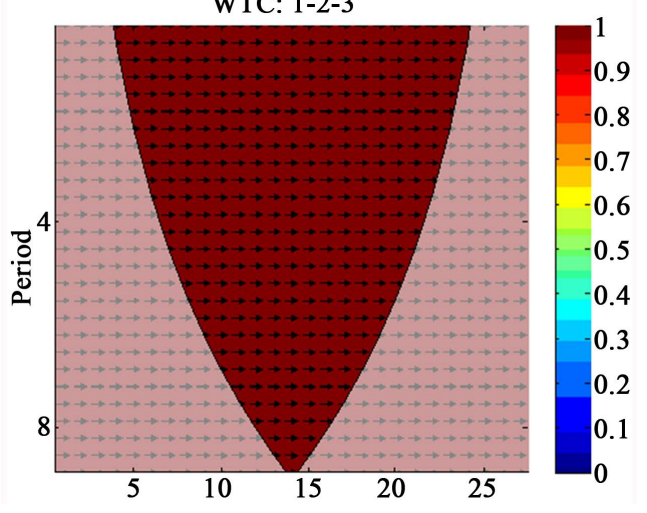

(c)

Figure 9. Wavelet coherence for the experimental data for $p=0.9$ and $q=3$.

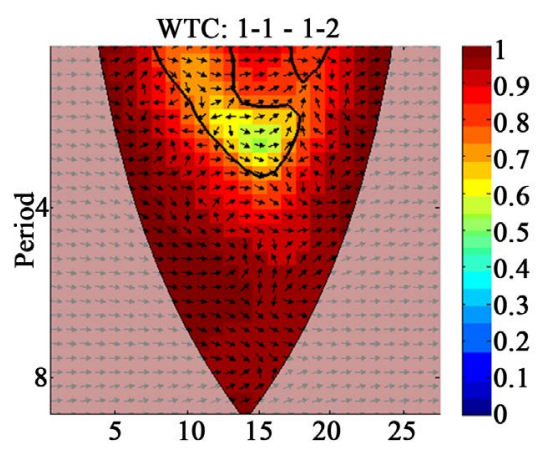

(a)

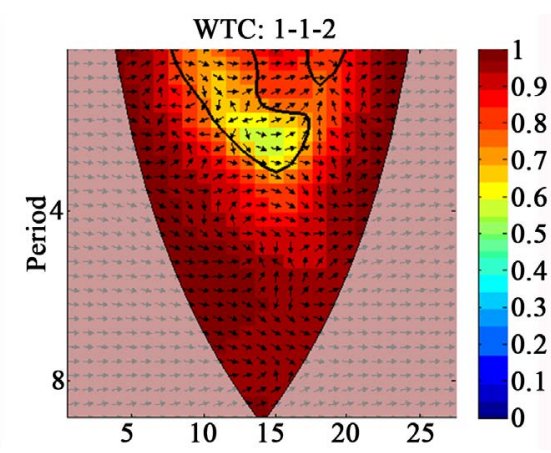

(b)

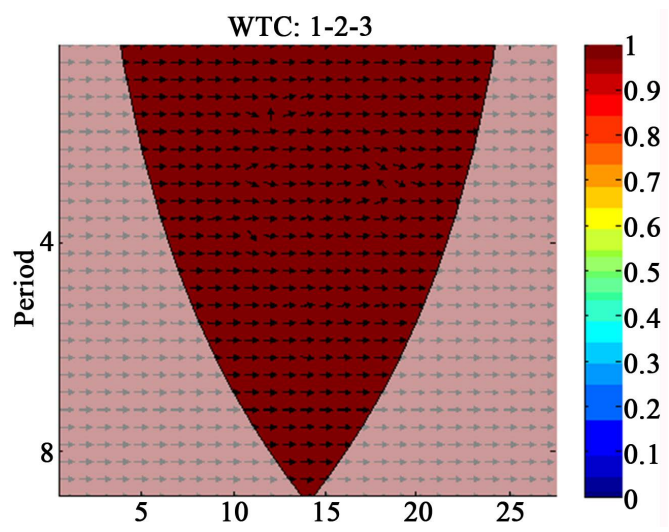

(c)

Figure 10. Wavelet coherence for the experimental data for $p=1.2$ and $q=3$. 


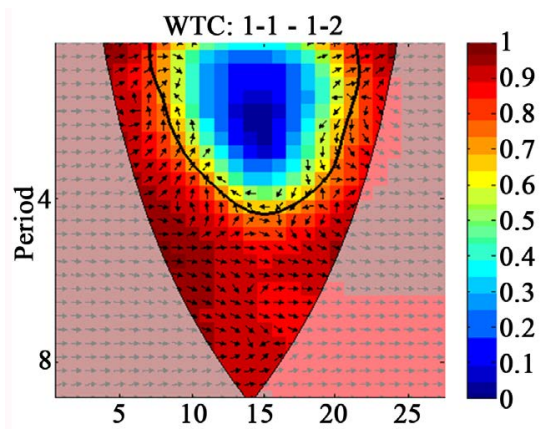

(a)

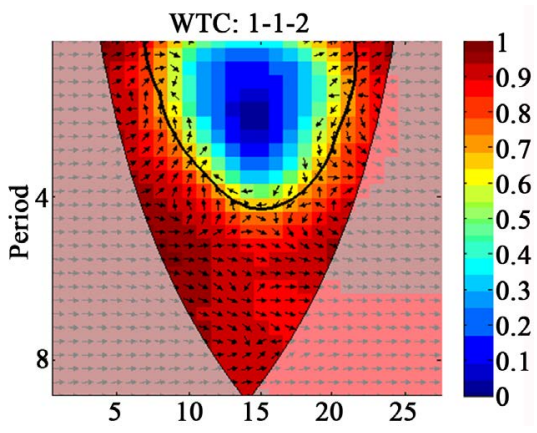

(b)

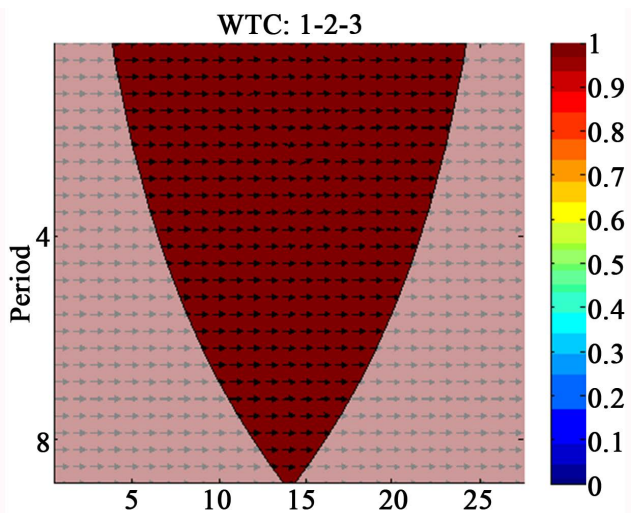

(c)

Figure 11. Wavelet coherence for the experimental data for $p=1.4$ and $q=3$.

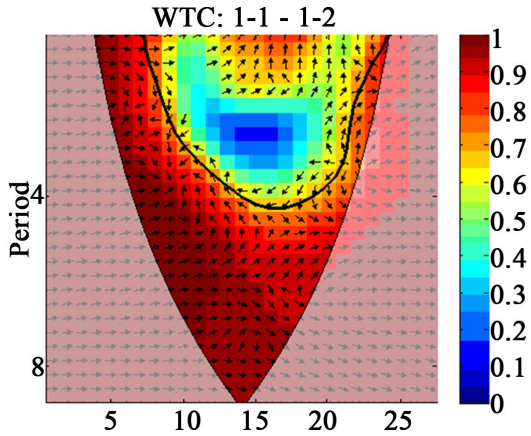

(a)

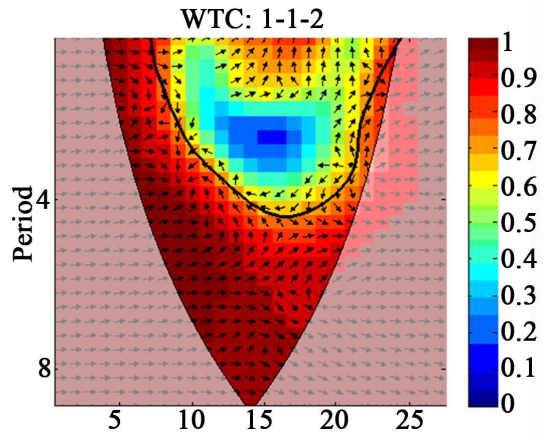

(b)

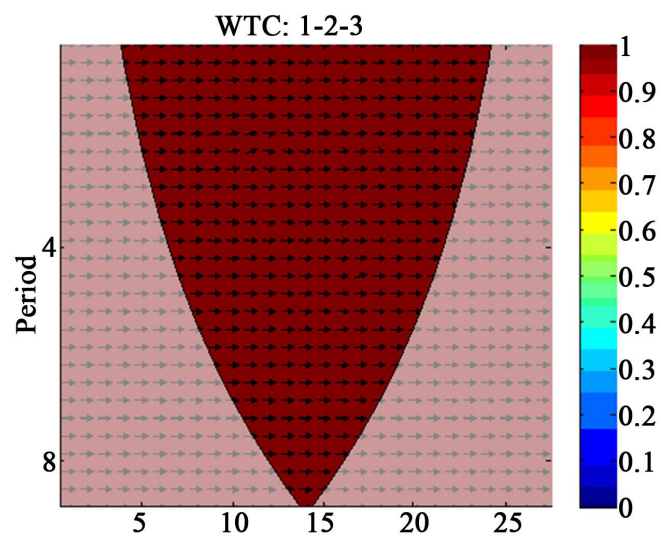

(c)

Figure 12. Wavelet coherence for the experimental data for $p=1.6$ and $q=3$. 


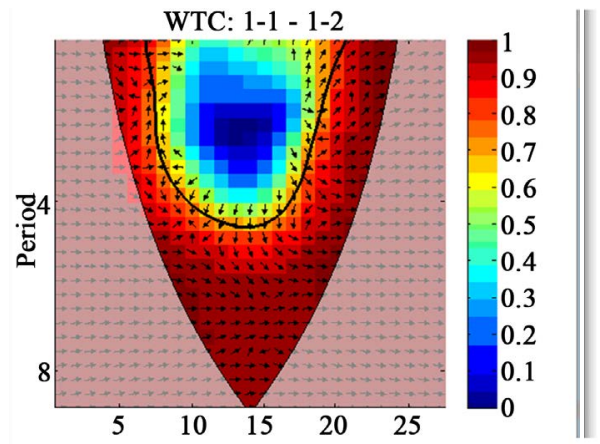

(a)

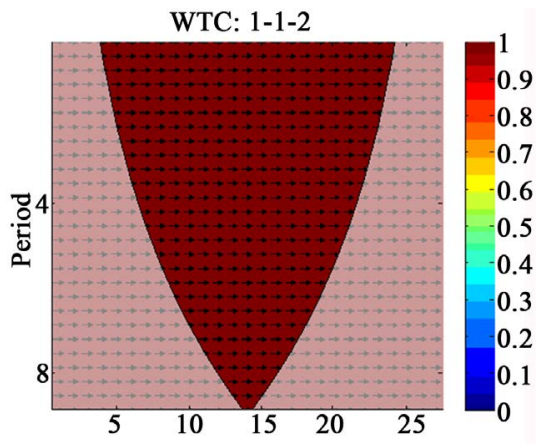

(b)

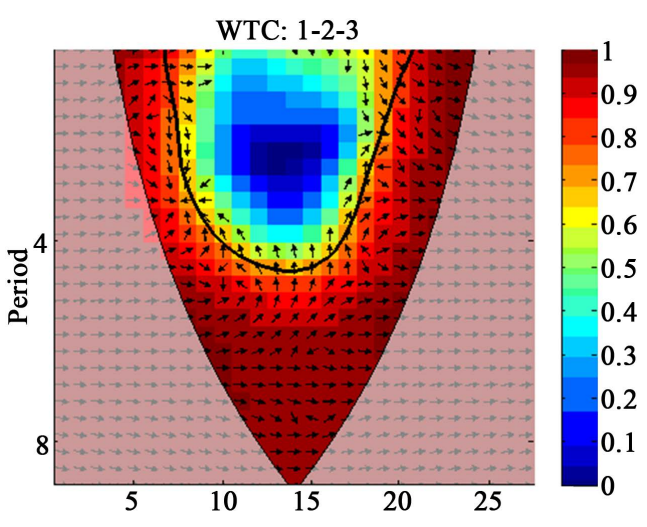

(c)

Figure 13. Wavelet coherence for the experimental data for $p=0.5$ and $q=4$.

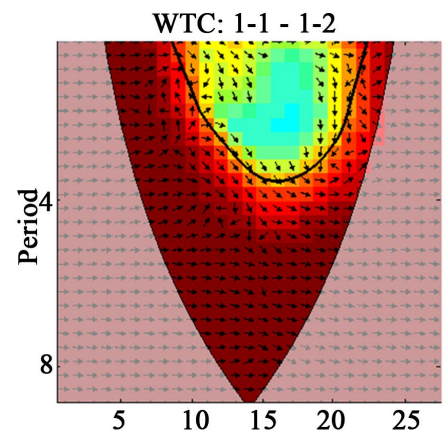

(a)

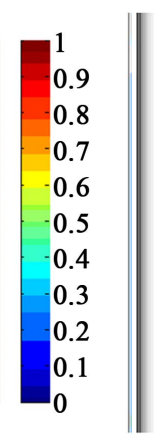

WTC: $1-2-3$

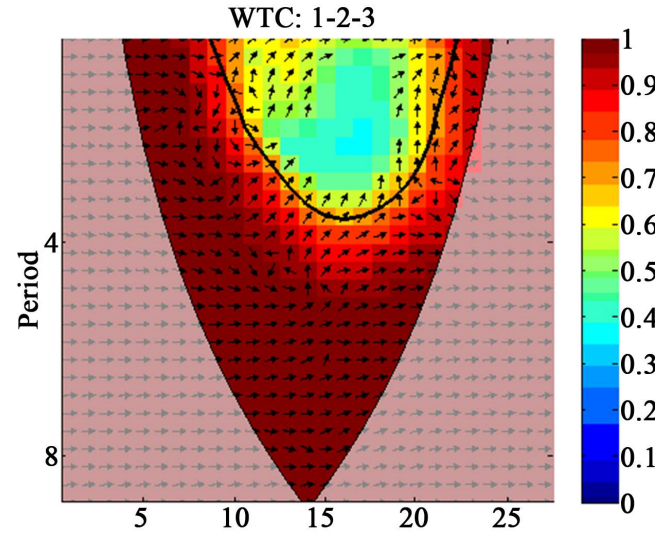

(c)

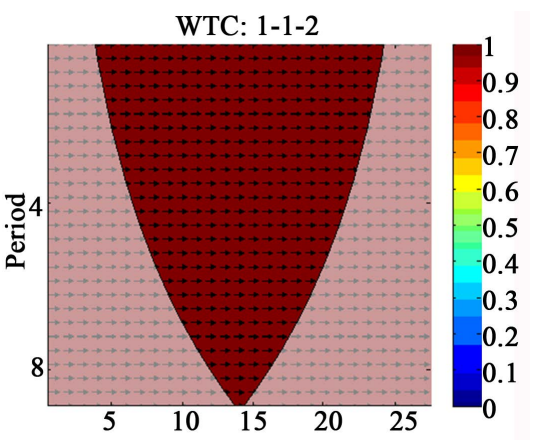

(b) 


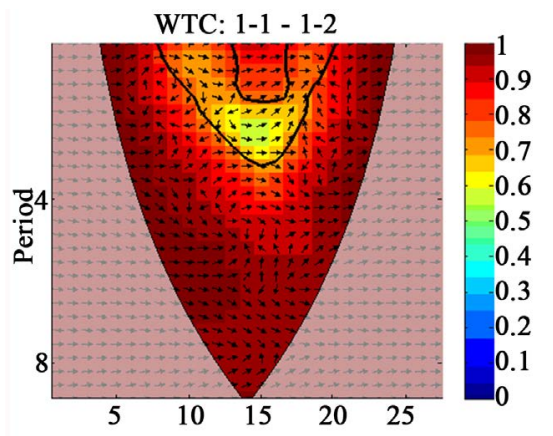

(a)

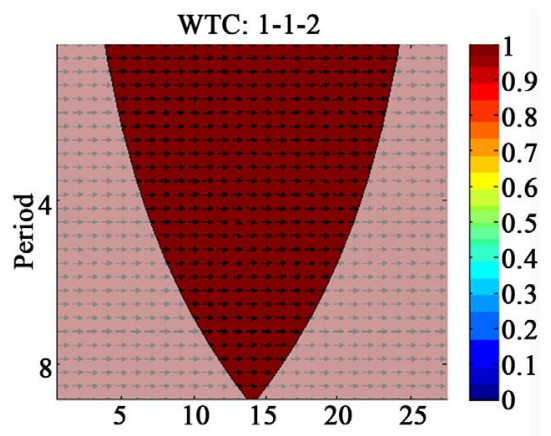

(b)

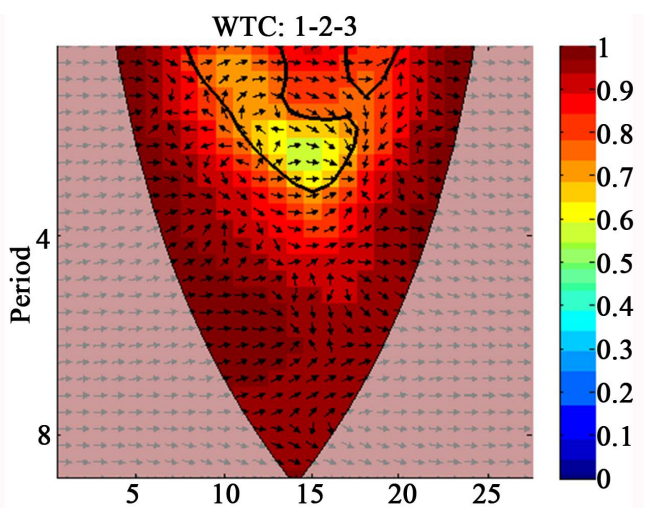

(c)

Figure 15. Wavelet coherence for the experimental data for $p=1.2$ and $q=4$.

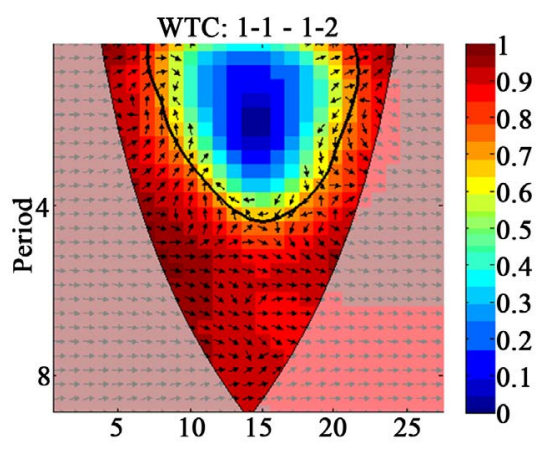

(a)

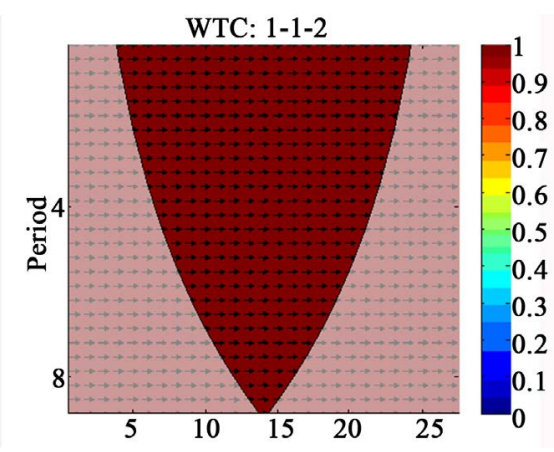

(b)

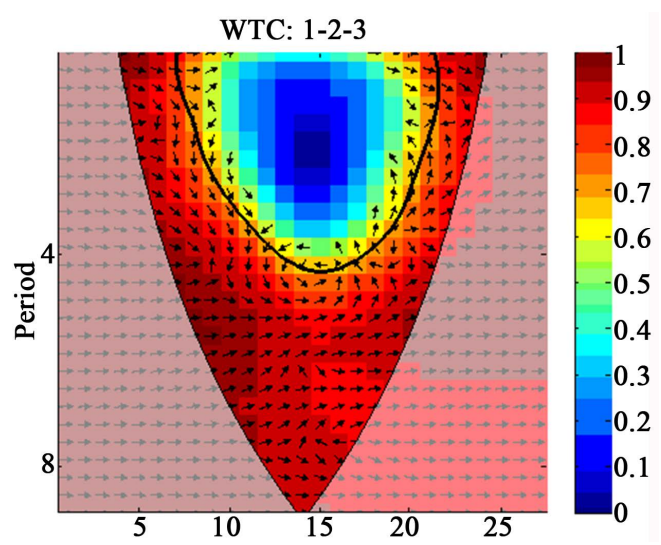

(c)

Figure 16. Wavelet coherence for the experimental data for $p=1.4$ and $q=4$. 


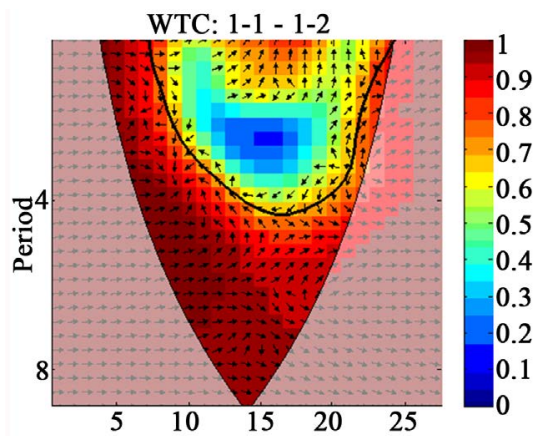

(a)

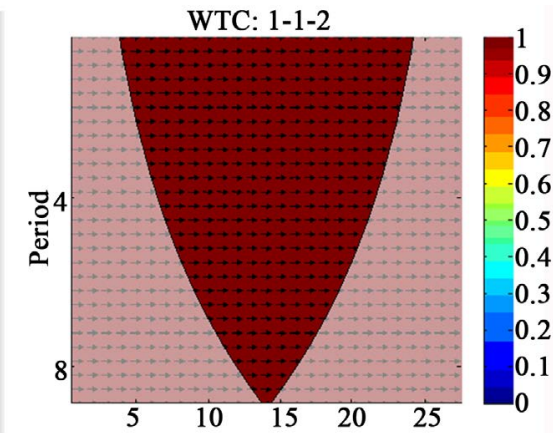

(b)

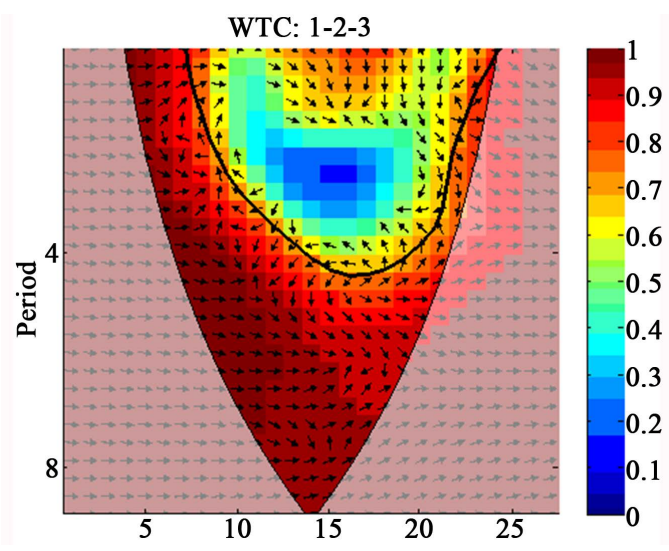

(c)

Figure 17. Wavelet coherence for the experimental data for $p=1.6$ and $q=4$.

areas delimited by the black lines cover coherence values significant at the 5\% level. In general, each point of wavelet reflects and clearly shown in Figure 3-17. One can measured their values in the time-frequency space, and it is calculated through wavelet transformation. The phase difference, indicated by arrows, that gives us details about delays of oscillation of the two examined time series. Arrows pointing to the right (left) when the time series are in-phase (anti-phase) or are positively (negatively) correlated. Arrow pointing up means that the first time series leads the second one, arrow pointing down indicates that the second time series leads the first one. In our case, these data suggest interrelations between different spaces ( $\eta$-space, $\phi$-space (in 1D) and $\eta \phi$-space (in 2D) respectively) and the impact of this relationship on the chaotic behavior in relativistic heavy ion collisions. Thus this implies that wavelet plots enable to appropriately identify both frequency bands and time intervals where data series move together.

The Figures 3-7 show the results of wavelet coherence for $q=2$. We see that between $\phi$-space and $\eta \phi$-space there is full consistency of the chaotic behavior in relativistic heavy ion collisions. Between $\eta$-space and $\phi$-space and between $\eta$-space and $\eta \phi$-space wavelet coherence varies depending on the value $\ln (M)$.

Thus, of the chaotic behavior in relativistic heavy ion collisions from the point of view of different spaces is changing. The smallest values the wavelet coherence is observed for the mean values $\ln (M)$. With increasing values $p$, violation of 
wavelet coherence to first decreases (Figures 3-5) and then increases (Figure 6 and Figure 7).

Figures 8-12 shows the results of wavelet coherence for $q=3$.We see that Figures 3-7 coincide with Figures 8-12. But we can to observe and minor differences (Figure 6 and Figure 11). It is connected with an existing fluctuation of the original data (Figure 6). At the same time it confirms the validity of the results that were obtained. In general, can be seen that with increasing $q$ (from $q=$ 2 to $q=3$ ) significant changes of the chaotic behavior in relativistic heavy ion collisions between different phase spaces does not occur.

Figures 13-17 shows the results of wavelet coherence for $q=4$.

We see that between $\eta$-space and $\eta \phi$-space there is full consistency of the chaotic behavior in relativistic heavy ion collisions. Between $\eta$-space and $\phi$-space and between $\phi$-space and $\eta \phi$-space wavelet coherence varies depending on the value $\ln (M)$. Other characteristics of the wavelet coherency have not changed. Thus, with increasing $\mathrm{q}$ (from $q=3$ to $q=4$ ) we can observe changes in the interaction phase spaces in the study of the chaotic behavior in relativistic heavy ion collisions. Therefore with the change in value of $\mathrm{q}$ there is a change influence of the chaotic behavior in relativistic heavy ion collisions, which is reflected in the change the values $C_{p, q}(M)$ of in the phase space.

\section{Conclusions and Final Remark}

The relativistic heavy ion collisions provide an experimental setting for the study of the exotic behavior of the matter. It has been suggested that the strongly interacting matter at high energy densities produced in these collisions may undergo a phase transition to quark gluon plasma (QGP). The produced particles in such nuclear collisions are believed to carry relevant information about the collision mechanism.

We have received different wavelet coherency for the chaotic behavior in relativistic heavy ion collisions between different phase spaces $\eta$-space, $\phi$-space (in one dimension) and $\eta \phi$-space (in two dimensions) respectively). We have shown changes in the wavelet coherence when changing the values of $q$ and $p$. This must be considered when analyzing the data.

The ideology of wavelet analysis and/or methodology was found an important tool for the wavelet coherency between the studied data series and it can be implemented very well for the study of relativistic heavy ion collisions.

\section{Acknowledgements}

The authors would like to acknowledge the keen support for this work of the Department of Physics, Faculty of Science, University of Tabuk, Saudi Arabia and also the Department of Informatics, Kharkiv National University of RadioElectronics, Kharkiv, Ukraine.

\section{References}

[1] Gyulassy, M. (1984) Signatures of New Phenomena in Ultrarelativistic Nuclear Col- 
lisions. Nuclear Physics A, 418, 59-85.

[2] Stephanov, M., Rajagopal, K. and Shuryak, E. (1999) Event-by-Event Fluctuations in Heavy Ion Collisions and the QCD Critical Point. Physical Review D, 60, Article ID: 114028.

[3] Bjorken, J.D. (1983) Highly Relativistic Nucleus-Nucleus Collisions: The Central Rapidity Region. Physical Review D, 27, 140-151. https://doi.org/10.1103/physrevd.27.140

[4] Bass, S.A., et al. (1998) Microscopic Models for Ultrarelativistic Heavy Ion Collisions. Progress in Particle and Nuclear Physics, 41, 255-369.

[5] Ahmad, S. and Ahmad, M.A. (2006) A Comparative Study of Multifractal Moments in Relativistic Heavy-Ion Collisions. Journal of Physics G Nuclear and Particle Physics, 32, 1279-1293. https://doi.org/10.1088/0954-3899/32/9/006

[6] Ahmad, M.A. (2010) A Study of Intermittency and Multifractality in ${ }^{28} \mathrm{Si}$-Emulsion Collisions at 14.6A GeV. PhD Thesis, Aligarh Muslim University, Aligarh.

[7] Chernov, G.M., et al. (1977) Central Collisions Produced by Relativistic Heavy Ions in Nuclear Emulsion. Nuclear Physics A, 280, 478-490.

[8] Adamovich, M.I., et al. (1991) Slow Target Associated Particles Produced in Ultra-Relativistic Heavy Ion Interactions. Physics Letters B, 262, 369-374.

[9] Mohery, M. and Abd-Allah, N.N. (2002) Systematic Comparison of the Experimental Data with the Fritiof Model in Nucleus-Nucleus Interactions with Light and Heavy Target Nuclei in Nuclear Emulsion at $4.5 \mathrm{~A} \mathrm{GeV/c}$. International Journal of Modern Physics E, 11, 161-175. https://doi.org/10.1142/S0218301302000776

[10] Mohery, M. (2012) Characteristics of the Total Disintegration Events of Emulsion Heavy Target Nuclei Caused by O16 and 28Si Nuclei at High Energies. Canadian Journal of Physics, 90, 1267-1278. https://doi.org/10.1139/p2012-104

[11] Jilany, M.A. (2004) Nuclear Fragmentation in Interactions of $3.7 \mathrm{~A} \mathrm{GeV} \mathrm{Mg} 24$ Projectiles with Emulsion Targets. Physical Review C, 70, 014901-014912. https://doi.org/10.1103/PhysRevC.70.014901

[12] Andersson, B., Gustafson, G. and Nilsson-Almqvist, B. (1987) A Model for Low- $\mathrm{p}_{\mathrm{T}}$ Hadronic Reactions with Generalizations to Hadron-Nucleus and Nucleus-Nucleus Collisions. Nuclear Physics B, 281, 289-309.

[13] Nilson-Almquist, B. and Stenlund, E. (1987) Interaction between Hadrons and Nuclei: The Lund Monte Carlo-Frtitiof Version 1.6. Computer Physics Communications, 43, 387-397.

[14] Demelford, E.A., Dremin, I.M. and Kittel, W. (1996) Scaling Laws for Density Correlations and Fluctuations in Multiparticle Dynamics. Physics Report, 1, 1270.

[15] Lyashenko, V., Kobylin, O. and Ahmad, M.A. (2014) General Methodology for Implementation of Image Normalization Procedure Using Its Wavelet Transform. International Journal of Science and Research, 3, 2870-2877.

[16] Lyashenko, V.V., Ayaz Ahmad, M. and Kobylin, O. (2016) Wavelet Analysis as a Learning Tool a Polymer Composites. American Journal of Engineering Research, 6, 1-6.

[17] Ahmad, S., Ayaz Ahmad, M., Irfan, M. and Zafar, M. (2006) Study of Non-Statistical Fluctuations in Relativistic Nuclear Collisions. Journal of the Physical Society of Japan, 75, Article ID: 064604. https://doi.org/10.1143/jpsj.75.064604

[18] Ahmad, S. and Ayaz Ahmad, M. (2006) Some Observations Related to Intermittency and Multifractality in ${ }^{28} \mathrm{Si}$ and ${ }^{12} \mathrm{C}$-Nucleus Collisions at $4.5 \mathrm{~A} \mathrm{GeV}$. Nuclear Physics A, 780, 206-221. 
[19] Ahmad, S. and Ayaz Ahmad, M. (2007) Study of the Levy Stability and Intermittent Behavior in ${ }^{28} \mathrm{Si}$-Emulsion Collisions at 4.5A GeV. Nuclear Physics A, 789, 298-310.

[20] Tariq, M., Ayaz Ahmad, M., Ahmad, S. and Zafar, M. (2007) Analysis of High $\mathrm{N}_{\mathrm{s}}$-Multiplicity Events Produced in Relativistic Heavy Ion Collisions at $4.5 \mathrm{~A} \mathrm{GeV/c}$. Romanian Reports in Physics, 59, 773-790.

[21] Ayaz Ahmad, M. and Ahmad, S. (2007) Study of Non-Thermal Phase Transition in ${ }^{28} \mathrm{Si}-\mathrm{Nucleus}$ Collisions at $14.6 \mathrm{~A} \mathrm{GeV}$. International Journal of Modern Physics E, 7-8, 2241-2247.

[22] Ahmad, S., Ayaz Ahmad, M., Tariq, M. and Zafar, M. (2009) Charged Multiplicity Distribution of Relativistic Charged Particles in Heavy Ion Collisions. International Journal of Modern Physics E, 18, 1929-1944. https://doi.org/10.1142/S0218301309013968

[23] Ayaz Ahmad, M., Ahmad, S. and Zafar, M. (2010) Intermittent and Scaling Behaviour of Shower Particles Produced in the Collisions of ${ }^{28} \mathrm{Si}-\mathrm{Em}$ at $14.6 \mathrm{~A} \mathrm{GeV}$. Indian Journal of Physics, 84, 1675-1681. https://doi.org/10.1007/s12648-010-0156-2

[24] Ayaz Ahmad, M. and Ahmad, S. (2012) Study of Angular Distribution and KNO Scaling in the Collisions of ${ }^{28} \mathrm{Si}$ with Emulsion Nuclei at $14.6 \mathrm{~A} \mathrm{GeV}$. Ukrainian Journal of Physics, 57, 1205-1213.

[25] Ayaz Ahmad, M. and Baker, J.H. (2016) The Dependence of Average Multiplicity of Produced Charged Particles on Interacting Projectile Nucleons in Nuclear Collisions. Dialogo Journal, 3, 219-225.

[26] Ayaz Ahmad, M. (2016) Some Aspects of Multi-Particle Productions in Relativistic Nuclear Collisions. Dialogo Journal, 3, 255-262.

[27] Kingsbury, N. (1999) Image Processing with Complex Wavelets. Philosophical Transactions of the Royal Society of London A: Mathematical, Physical and Engineering Sciences, 357, 2543-2560. https://doi.org/10.1098/rsta.1999.0447

[28] Heil, C.E. and Walnut, D.F. (1989) Continuous and Discrete Wavelet Transforms. SIAM Review, 31, 628-666. https://doi.org/10.1137/1031129

[29] Delbeke, L. and Abry, P. (2000) Stochastic Integral Representation and Properties of the Wavelet Coefficients of Linear Fractional Stable Motion. Stochastic Processes and Their Applications, 86, 177-182.

[30] Veitch, D., Flandrin, P., Abry, P., Riedi, R. and Baraniuk, R. (2002) The Multiscale Nature of Network Traffic: Discovery, Analysis, and Modelling. IEEE Signal Processing Magazine, 19, 28-46. https://doi.org/10.1109/79.998080

[31] Torrence, C. and Webster, P.J. (1999) Interdecadal Changes in the ENSO-Monsoon System. Journal of Climate, 12, 2679-2690. https://doi.org/10.1175/1520-0442(1999)012<2679:ICITEM>2.0.CO;2

[32] Grinsted, A., Moore, J.C. and Jevrejeva, S. (2004) Application of the Cross Wavelet Transform and Wavelet Coherence to Geophysical Time Series. Nonlinear Processes in Geophysics, 11, 561-566. https://doi.org/10.5194/npg-11-561-2004

[33] Bialas, A. and Gradzicki, M. (1990) A New Variable to Study Intermittency. Physics Letters B, 252, 483-486.

[34] Bialas, A. and Peschanski, R. (1986) Moments of Rapidity Distributions as a Measure of Short-Range Fluctuations in High-Energy Collisions. Nuclear Physics B, 273, 703-718.

[35] Bialas, A. and Peschanski, R. (1988) Intermittency in Multiparticle Production at High Energy. Nuclear Physics, 308, 857-867.

[36] Hwa, R.C. (1990) Fractal Measures in Multiparticle Production. Physical Review D, 
41, 1456-1462. https://doi.org/10.1103/PhysRevD.41.1456

[37] Hwa, R.C. (1996) Criticality, Erraticity and Chaoticity in Strong Interaction. Proceedings of 7 th International Workshop on Multiparticle Production, Correlation and Fluctuations, Nijmegen, 302.

[38] Hwa, R.C. and Cao, Z. (1997) Fluctuations of Spatial Patterns as a Measure of Classical Chaos. Physical Review E, 56, 326-333. https://doi.org/10.1103/PhysRevE.56.326

[39] Hwa, R.C. and Zhang, Q. (2000) Fluctuation of Gaps in Hadronization at Phase Transition. Physical Review D, 62, 014003-014009.

[40] Ayaz Ahmad, M., Rasool, M.H., Ahmad, S., Ahmad, N.A. and Madani, J.H. (2013) Chaotic Behaviour of Multiparticle Production in Relativistic Heavy Ion Collisions. International Journal of Enhanced Research in Science Technology \& Engineering, 2, 77-89.

[41] Wang, S. and Wang, Z. (1998) Chaotic Behavior of Multiparticle Production in p-p Collisions at 400GeV/c. Physical Review D, 57, 3036. https://doi.org/10.1103/PhysRevD.57.3036

[42] Fu, J., Wu, Y. and Liu, L. (2000) The Influence of Statistical Fluctuations on the Erraticity Behavior of Multiparticle System. Physics Letters B, 472, 161-167.

Submit or recommend next manuscript to SCIRP and we will provide best service for you:

Accepting pre-submission inquiries through Email, Facebook, LinkedIn, Twitter, etc. A wide selection of journals (inclusive of 9 subjects, more than 200 journals)

Providing 24-hour high-quality service

User-friendly online submission system

Fair and swift peer-review system

Efficient typesetting and proofreading procedure

Display of the result of downloads and visits, as well as the number of cited articles

Maximum dissemination of your research work

Submit your manuscript at: http://papersubmission.scirp.org/

Or contact jamp@scirp.org 